\title{
Analysis of kinase gene expression in the frontal cortex of suicide victims: implications of fear and stress $^{\dagger}$
}

\section{Kwang Choi*, Thien Le, Guoqiang Xing, Luke R. Johnson and Robert J. Ursano}

Department of Psychiatry, Center for the Study of Traumatic Stress, Uniformed Services University of Health Sciences, Bethesda, MD, USA

\section{Edited by:}

Regina M. Sullivan, Nathan Kline Institute and NYU School of Medicine, USA

\section{Reviewed by:}

Fabrício A. Pamplona, Universidade

Federal de Santa Catarina, Brazil Michael R. Bruchas, Washington

University in St. Louis, USA

\section{${ }^{*}$ Correspondence:}

Kwang Choi, Department of Psychiatry, Uniformed Services University of Health Sciences, 4301 Jones Bridge Road, Bethesda, MD 20814, USA. e-mail: kwang.choi@usuhs.mil

${ }^{+}$The views expressed in this article are those of the authors and do not necessarily reflect the official policy or position of the Department of Defense, nor the U.S. Government.
Suicide is a serious public health issue that results from an interaction between multiple risk factors including individual vulnerabilities to complex feelings of hopelessness, fear, and stress. Although kinase genes have been implicated in fear and stress, including the consolidation and extinction of fearful memories, expression profiles of those genes in the brain of suicide victims are less clear. Using gene expression microarray data from the Online Stanley Genomics Database $^{1}$ and a quantitative PCR, we investigated the expression profiles of multiple kinase genes including the calcium calmodulin-dependent kinase (CAMK), the cyclin-dependent kinase, the mitogen-activated protein kinase (MAPK), and the protein kinase $\mathrm{C}(P K C)$ in the prefrontal cortex (PFC) of mood disorder patients died with suicide $(N=45)$ and without suicide $(N=38)$. We also investigated the expression pattern of the same genes in the PFC of developing humans ranging in age from birth to 49 year $(N=46)$. The expression levels of CAMK2B, CDK5, MAPK9, and $P R K C /$ were increased in the PFC of suicide victims as compared to non-suicide controls (false discovery rate, FDR-adjusted $p<0.05$, fold change $>1.1$ ). Those genes also showed changes in expression pattern during the postnatal development (FDR-adjusted $p<0.05$ ). These results suggest that multiple kinase genes undergo age-dependent changes in normal brains as well as pathological changes in suicide brains. These findings may provide an important link to protein kinases known to be important for the development of fear memory, stress associated neural plasticity, and up-regulation in the PFC of suicide victims. More research is needed to better understand the functional role of these kinase genes that may be associated with the pathophysiology of suicide.

Keywords: brain development, frontal cortex, posttraumatic stress disorder, anxiety disorder, psychiatric genomics, bioinformatics

\section{INTRODUCTION}

Physical and mental threat can induce fear responses, and fear can be associated with objects and places through a process of Pavlovian fear conditioning (Ledoux, 2000). The process of fear learning and, importantly, its "overriding" or fear extinction is dependent on the amygdala and the prefrontal cortex (PFC). Stress is a multi-dimensional challenge to physical and mental homeostasis that can be triggered by fear (Kim and Diamond, 2002; McEwen, 2007), and both acute and chronic stress can alter the properties of fear (Conrad et al., 1999; Rodrigues et al., 2009). A growing body of evidence suggests that fear and traumatic stress may contribute to the pathophysiology of suicide. For instance, suicidal ideation was significantly associated with traumatic life events and the effects of traumatic stress on suicidal behavior may be mediated by feelings of hopelessness (Tarrier and Picken, 2010; Guerra and Calhoun, 2011).A study using a large scale of civilian population $(N=34,653)$ found that over $70 \%$ of the individuals who reported a lifetime history of a suicide attempt had anxiety disorders (Nepon et al., 2010). Interestingly, individuals with comorbidity of personality disorders such as neuroticism and posttraumatic stress disorder (PTSD) showed a much stronger association with suicide attempts than those who had PTSD alone. In a military population, more U.S. service members have

${ }^{1}$ www.stanleygenomics.org been deployed since September 2001 than in the previous 40 years. A greater number of these deployed service members are surviving, which has increased the incidence of combat-related PTSD among those veterans (Callahan, 2010). For example, Iraq and Afghanistan War veterans who showed PTSD symptoms were four times more likely to endorse suicidal ideation than their non-PTSD counterparts (Jakupcak et al., 2009). These evidences suggest that fearful memories and PTSD symptoms may contribute to suicidal ideation and attempts, and individual vulnerability to traumatic events is one of the risk factors for suicide (Ursano et al., 2010). However, precise molecular mechanisms underlying how fear and stress trigger suicidal behavior in humans are not clearly understood.

Multiple brain regions have been implicated in emotional learning and memory. Among those, the PFC is one of the key brain regions that integrates stress signals and subsequent decision-making process in humans. Protein phosphorylation in the brain plays a critical role in triggering synaptic changes that are associated with emotional learning and memory (Fischer et al., 2003). Intracellular phosphorylation is orchestrated by a complex network of many different kinases including protein kinase $\mathrm{C}(P K C)$, calcium/calmodulin-dependent protein kinase $(C A M K)$, mitogen-activated protein kinase (MAPK), and cyclin-dependent kinase $(C D K)$. $P K C$ is a critical phosphorylating enzyme in the phosphoinositide signaling pathway. Previous 
studies have suggested that these kinases such as $P K C, C A M K$, $M A P K$, and $C D K$ also regulate fear conditioning and extinction in animals (Schafe et al., 2000; Li et al., 2002; Frankland et al., 2004; Lepicard et al., 2006; Sananbenesi et al., 2007; Bergstrom et al., 2011). For example, chronic administration of a $P K C$ inhibitor reduced the acquisition of conditioned fear memory suggesting the involvement of $P K C$ in the synaptic plasticity and memory (Li et al., 2002). On the contrary, another study reported that inhibition of $P K C$ signaling protected dendritic spines in the PFC and rescued working memory impairment caused by chronic stress (Hains et al., 2009). The authors suggested that $P K C$ inhibitors may act as neuroprotective agents in fear and stress-related disorders. $C A M K$ is also involved in hippocampaldependent contextual learning in rodents (Kouzu et al., 2000). Among the individuals with psychiatric disorders, the expression levels of CaMKII beta (CAMK2B) were increased in the PFC of individuals with schizophrenia $(27 \%)$ and individuals with depression (36\%) as compared to the unaffected controls (Novak et al., 2006). Because CAMK2B regulates important functions in the brain such as neurotransmitter signaling, neural outgrowth, and pruning, its increased expression in the PFC of the individuals with psychiatric disorders may have important implications. Moreover, inhibition of the MAPK pathway in the hippocampus abolished the increased contextual fear conditioning induced by glucocorticoids in mice (Revest et al., 2005). This suggests that the MAPK pathway interacts with the glucocorticoid system in fear learning and memory. Another kinase, cyclin-dependent kinase 5 (CDK5), has been implicated in learning and memory (Fischer et al., 2003). CDK5 plays a role during neurodevelopmental processes, such as interactions with distinct cytoplasmic and synaptic target molecules, and synaptic plasticity underlying memory consolidation in the adult brain. It has been shown that extinction of fear memory requires down-regulation of CDK5 activity in the mouse hippocampus (Sananbenesi et al., 2007). Taken together, these studies indicate that multiple kinase systems contribute to regulation of emotional learning and memory, and subsequent behavioral responses in animals and in humans.

Recent advances in genomic technologies utilizing postmortem brain tissue have made significant progress toward more analytical and informative research in psychiatry (Harrison, 2011; Mccullumsmith and Meador-Woodruff, 2011). However, identifying potential susceptibility genes associated with suicide has been challenging (Mann et al., 2009; Tsai et al., 2011). We have shown that gene expression changes in postmortem brain tissue are subtle possibly due to a relatively small sample size, known and unknown confounding factors, and diagnostic heterogeneity among psychiatric patients (Choi et al., 2008). Given that multiple kinase genes are implicated in the mechanisms of fear and stress, and fear and stress are the major risk factors for suicidal behavior, we hypothesized that the kinases genes may be involved in the pathophysiology of suicidal behavior. Thus, we investigated the expression profiles of four major kinase genes including protein kinase $\mathrm{C}(P K C)$, calcium/calmodulin-dependent protein kinase (CAMK), mitogenactivated protein kinase $(M A P K)$, and $C D K$ in the PFC of mood disorder patients died with and without suicide. In order to study the developmental expression pattern of the same genes, we measured mRNA levels in the PFC of normal individuals ranging in age from birth to 49 years. Using gene expression microarrays from the postmortem brain tissue, we aimed to identify potential kinase genes that are associated with both chronological age and suicide.

\section{MATERIALS AND METHODS POSTMORTEM BRAIN TISSUE}

Gene expression microarray datasets from the individuals with mood disorders with suicide $(N=45)$ and without suicide $(N=38)$ were obtained from the Stanley Online Genomics database (see text footnote 1). The details of the brain sample collection have been described previously (Torrey et al., 2000). Developmental brain tissue from the PFC of subjects $(N=46)$ ranging in age from 1 month to 49 years was obtained from the National Institute of Child Health and Human Development Brain and Tissue Bank for Developmental Disorders (UMBB; NICHHD contract\# NO1-HD8-3283). The collection protocol was reviewed and approved by the Institutional Review Board of the University of Maryland, Baltimore. For the developmental brains, all subjects were free of neurological and psychiatric symptoms at the time of death as described previously (Choi et al., 2009). Developmental brain microarray raw data are available from the gene expression omnibus (GEO) with an Accession number GSE11512.

\section{MICROARRAY EXPERIMENT}

Total RNA was extracted from gray matter of the PFC (BA 46) and using the Trizol method (Invitrogen, Carlsbad, CA, USA). Samples were included only if the RNA was of good quality (RNA integrity number, RIN > 7) as determined by the Bioanalyzer 2100 electrophoresis system (Agilent Technologies, Foster City, CA, USA). Purified RNA was carried through the Affymetrix preparation protocol ${ }^{2}$, and each sample was hybridized to the different Affymetrix platform such as HGU95av2, HGU 133a, HGU 133b, or HGU133 plus 2.0 GeneChip to assess genome-wide expression profiles. RNA processing and microarray data generation was performed by the individual investigators at their own facilities as described previously (Choi et al., 2008).

\section{QUALITY CONTROL OF MICROARRAYS}

Raw microarray data were processed and analyzed using the R statistical language ${ }^{3}$ and the Bioconductor packages (Gentleman et al., 2004). A robust multi-array average (RMA) algorithm was used for normalization of expression values (log base 2 ) for each transcript (Irizarry et al., 2003). Microarray data quality was assessed using a pair-wise sample correlation coefficient with hierarchical clustering. Transcripts were filtered out if $20 \%$ or more of the subjects had expression values of less than a 1.1-fold change in either direction from the transcript's median value and if the percent of subjects with an absent gene call exceeded 33\% using the Affymetrix calls.

\section{MICROARRAY ANALYSIS OF THE DEVELOPMENTAL BRAINS}

First, we analyzed individual demographic factors including brain $\mathrm{pH}$, postmortem interval (PMI), RIN, race, and sex to identify potential confounds affecting the expression of a significant number of genes $(p<0.001)$. Following the demographic variable analyses,

${ }^{2}$ www.Affymetrix.com

${ }^{3}$ http://www.r-project.org 
gene expression across chronological age was analyzed in a series of multiple regression models, one model for each gene, including age (log base 2 ) and brain $\mathrm{pH}$ as independent variables and gene expression (log base 2) as a dependent variable (Choi et al., 2009). To correct for multiple testing of the genes, the calculated $p$-values corresponding to the age covariate for each gene were adjusted to give an overall false discovery rate (FDR) of $5 \%$ using the $q$-value $(q \mathrm{v})$ package $\mathrm{p}^{4}$ The criteria of significance were set at $q \mathrm{v}<0.05$.

\section{MICROARRAY ANALYSIS OF MOOD DISORDER WITH AND WITHOUT SUICIDE}

Mood disorder subjects were divided into two groups: those with suicide $(N=45)$ and those without suicide $(N=38)$. Each pre- and post-mortem variable was compared between the suicide and the non-suicide group. We identified the variables including age, mood disorder, and duration of illness that were different between the two groups $(p<0.05)$. Thus, we adjusted for these variables in the following suicide analysis using multiple regression models. For an individual study analysis, we performed a linear regression analysis to calculate an adjusted fold change, SE, and $p$-value for each gene in each study. We then performed a cross-study comparison based on scaled representations of individual study-level analysis across nine microarray studies. Consensus fold change was calculated for each gene based on a weighted combination of the individual fold changes and the SEs for the microarray probe sets that map to each gene across the studies as described previously (Choi et al., 2008). Weights were determined in a probe set specific manner to account for the different levels of precision associated with each probe set that map to a given gene across the studies. The weights were equal to 1/SE $i$, where $\mathrm{SE} i$ is the SE of the $i$ th probe set for the gene across all the studies. To adjust for multiple testing of the genes, the calculated $p$-values corresponding to the suicide group were adjusted to give an overall FDR of 5\% using the qv package. The criteria of significance were set at $q \mathrm{v}<0.05$ and fold change $>1.1$.

\section{BIOINFORMATICS MAPPINGS}

The NCBI's Database for Annotation, Visualization, and Integrated Discovery was used as the standard source for gene annotation information [22]. The primary fields extracted from the DAVID include: Entrez ID, gene symbol, gene name, and gene summary. For the microarrays, queries were based on the Affymetrix probe set ID (AFFYID).

\section{RESULTS}

A summary of the subject characteristics included in the microarray studies is shown in Table 1. There were no significant differences in sex, race, brain $\mathrm{pH}$, and PMI between the suicide and the nonsuicide group; this is important since these pre- and post-mortem variables appear to influence gene expression in the postmortem brain tissue. The number of bipolar disorder subjects was slightly higher in the non-suicide group (66 vs. 51\%) while the number of major depression subjects was higher in the suicide group (49 vs. $34 \%)$. Other variables such as age (47.2 vs. 41.7$)$ and duration of illness (19.4 vs. 15) were different between the suicide and the

${ }^{4}$ www.bioconductor.org

${ }^{5}$ http://david.abcc.ncifcrf.gov/ non-suicide group $(p<0.05)$. Thus, we adjusted for age, mood disorder (bipolar disorder and major depression) and duration of illness in the following suicide analysis using a multiple regression model. Among the 45 suicide cases, a majority of the subjects died of drug overdose (36\%), hanging (29\%), jumped (11\%), and gun shot wound (9\%).

Figure 1 shows the expression profiles of $C A M K 2 B$ in the PFC of normal individuals ranging in age from birth to 49 years (A) and suicide victims as compared to the non-suicide subjects (B). The expression levels of $C A M K 2 B$ in the PFC were gradually decreased during the postnatal development $\left(r^{2}=0.69, q \mathrm{v}=1.1 \mathrm{E}\right.$ 11). Each subject was color-coded based on the arbitrary age group such as neonate (red), infant (green), toddler (blue), school age (magenta), teenage (pink), young adult (yellow), and adult (gray). A combined analysis of the microarray studies shows that $C A M K 2 B$ expression levels are significantly increased in the PFC of suicide victims as compared to the non-suicide subjects (FC: $1.13, q \mathrm{v}=0.009)$. There are multiple microarray probes $(N=2-5)$ for $C A M K 2 B$ in each study and those probes show consistent changes in expression levels. Although most of the individual studies did not show significant changes, the combined analysis (shown on the bottom) showed consensus increases in expression in the suicide group as compared to the non-suicide group.

The expression levels of CDK5 in the PFC during postnatal development show inverted U-shape (Figure 2A). Specifically, the expression levels of CDK5 in the PFC were increased until 10 years of age $\left(r^{2}=0.18, q \mathrm{v}=0.035\right)$ and then gradually decreased until 49 years $\left(r^{2}=0.23, q v=0.029\right)$. A combined analysis of the microarray studies shows that $C D K 5$ expression levels are increased in the PFC of suicide victims as compared to the non-suicide subjects (FC: $1.19, q \mathrm{v}=0.04$ ) as shown in Figure 2B. Individual studies did not show significant changes but a combined analysis (shown on the bottom) showed a significant up-regulation of CDK5 in the PFC of suicide victims.

Figure $3 \mathrm{~A}$ shows that the expression levels of mitogen-activated protein kinase 9 (MAPK9) in the PFC are gradually increased in the $\mathrm{PFC}$ of normal individuals ranging in age from birth to 49 years $\left(r^{2}=0.64, q \mathrm{v}=1.2 \mathrm{E}-05\right)$. The expression levels consistently increased

Table 1 |A summary of subject characteristics included in suicide microarray analysis.

\begin{tabular}{lll}
\hline & Non-suicide & Suicide \\
\hline Number of subjects & 38 & 45 \\
Age & $47.2(1.6)$ & $41.7(1.6)$ \\
Sex (male) & $55.3 \%$ & $55.6 \%$ \\
Race (caucasian) & $94.7 \%$ & $95.6 \%$ \\
Bipolar disorder & $65.8 \%$ & $51.1 \%$ \\
Major depression & $34.2 \%$ & $48.9 \%$ \\
PMl & $32.9(2.5)$ & $33.0(2.3)$ \\
Brain pH & $6.4(0.05)$ & $6.5(0.04)$ \\
Duration of illness & $19.4(1.7)$ & $15.0(1.4)$ \\
Lifetime antipsychotics & $11287(4021)$ & $4981(1707)$ \\
\hline
\end{tabular}

For each variable, mean \pm SE or percentage value is reported. PMI, postmortem interval. 


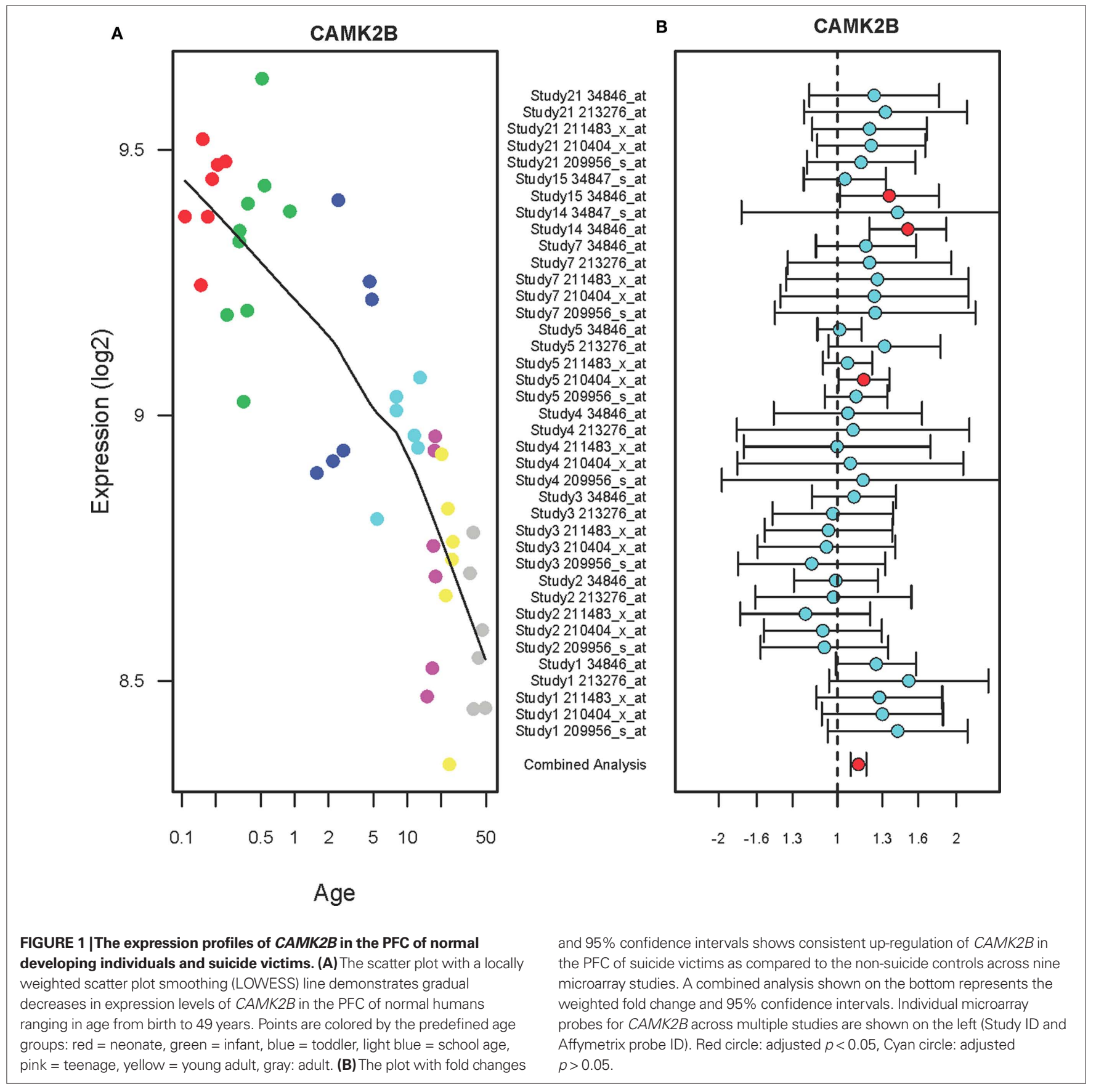

until 20 years of age then stabilized until 49 years. In the PFC of suicide victims, MAPK9 expression levels were increased as compared to the non-suicide subjects (FC: 1.21, $q \mathrm{v}=0.002$ ) as shown in Figure 3B. Each study has two microarray probes for MAPK9 and most of the probes showed a tendency toward increase in expression. A combined analysis of microarrays revealed increased expression levels of MAPK9 in the suicide victims as compared to the non-suicide controls.

A developmental expression pattern of the protein kinase $\mathrm{C}$ iota $(P R K C I)$ gene showed a gradual decrease in the PFC of normal individuals ranging in age from birth to 49 years $\left(r^{2}=0.66\right.$, $q \mathrm{v}=4.2 \mathrm{E}-06)$ as shown in Figure 4A. A combined analysis of the microarrays showed that $P R K C I$ expression levels were increased in the PFC of suicide victims as compared to the non-suicide subjects (FC: $1.14, q \mathrm{v}=0.015$ ) shown in Figure 4B. Each microarray study has three probes for PRKCI and two studies (Study ID 2 and 3 ) did not show any changes in PRKCI expression while the rest of studies showed a tendency toward increase in expression. A combined analysis of microarrays demonstrated small but consensus changes in expression levels of $P R K C I$ in the suicide group as compared to the non-suicide group. 


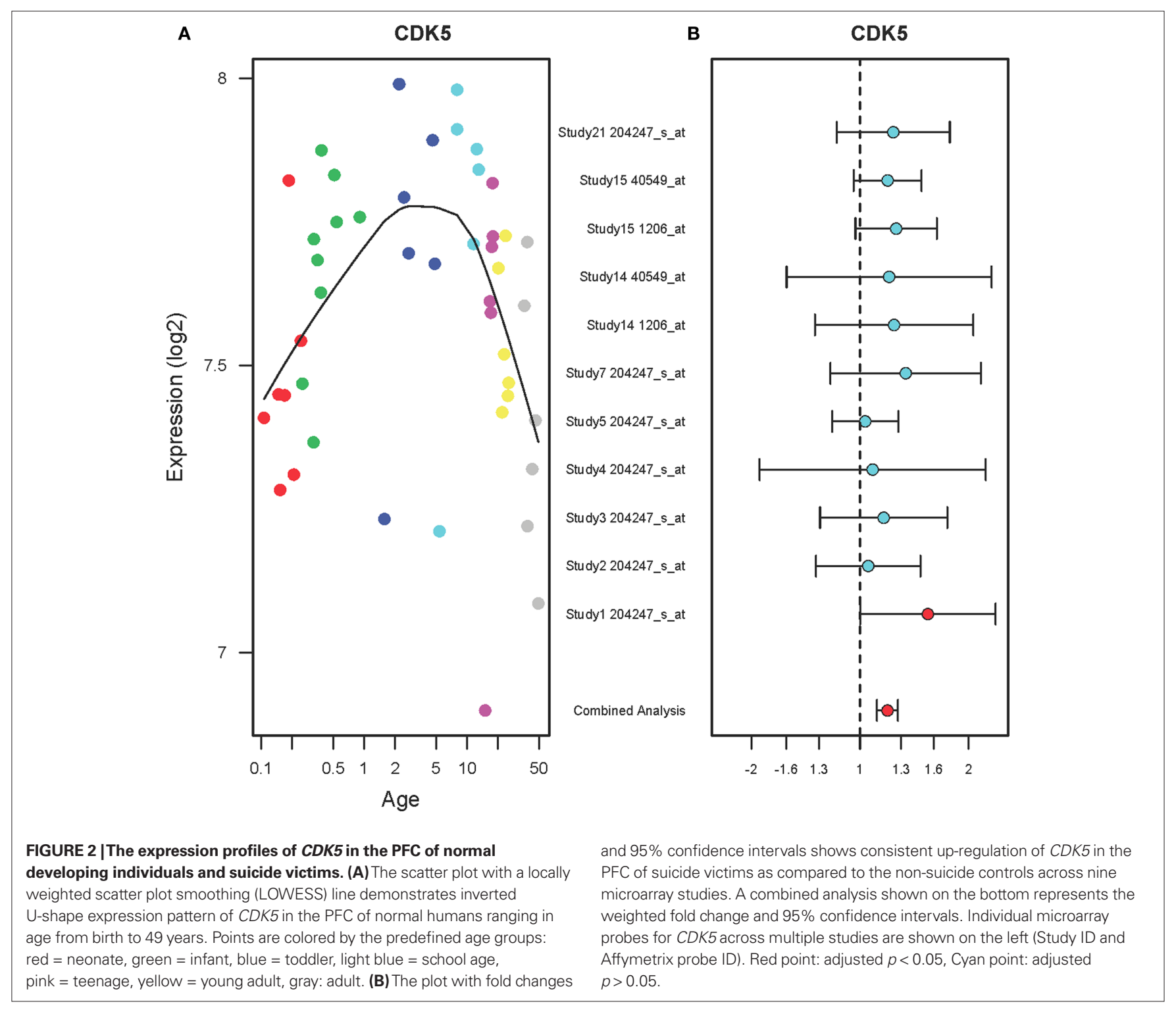

\section{DISCUSSION}

The mechanisms of suicide are complex mediated by the interaction of multiple factors including genes and environment (Brezo et al., 2008; Fiori et al., 2011). However, the search for specific genetic factors that contribute to the pathophysiology of suicide has been challenging (Mann et al., 2009; Tsai et al., 2011). Previous studies with rodent models with fear and stress suggested that kinase genes in the brain may play a role in fear and stress-related behavior (Kouzu et al., 2000; Li et al., 2002; Revest et al., 2005; Sananbenesi et al., 2007; Hains et al., 2009). Although fear and stress may contribute to suicidal behavior in humans, expression profiles of those kinases genes in suicide brains have not been well-characterized. Thus, we investigated the expression profiles of the kinase genes using the microarray data from a well-characterized cohort of postmortem brains of mood disorder patients who died with suicide and without suicide. We found that four kinase genes including CAMK2b,
CDK5, MAPK9, and PRKCI undergo age-dependent changes in expression in the PFC of normal individuals ranging in age from birth to 49 years. The expression levels of the same genes were increased in the PFC of suicide victims as compared to the nonsuicide controls. It is important to note that these genes have been implicated in the mechanisms of fear and stress-related disorders. Thus, these genes may also contribute to the pathophysiology of suicide via interactions with the fear and stress circuitry in the brain.

Here, we demonstrated the advantages of combining multiple microarray datasets to detect small but consensus changes in gene expression in the PFC of suicide victims. We found robust changes in gene expression in the PFC of normal individuals ranging in age from birth to 49 years, suggesting that chronological age is one of major factors affecting brain gene expression during development. We attempted to identify the genes that are associated with both chronological age and suicide phenotype in individuals with mood 


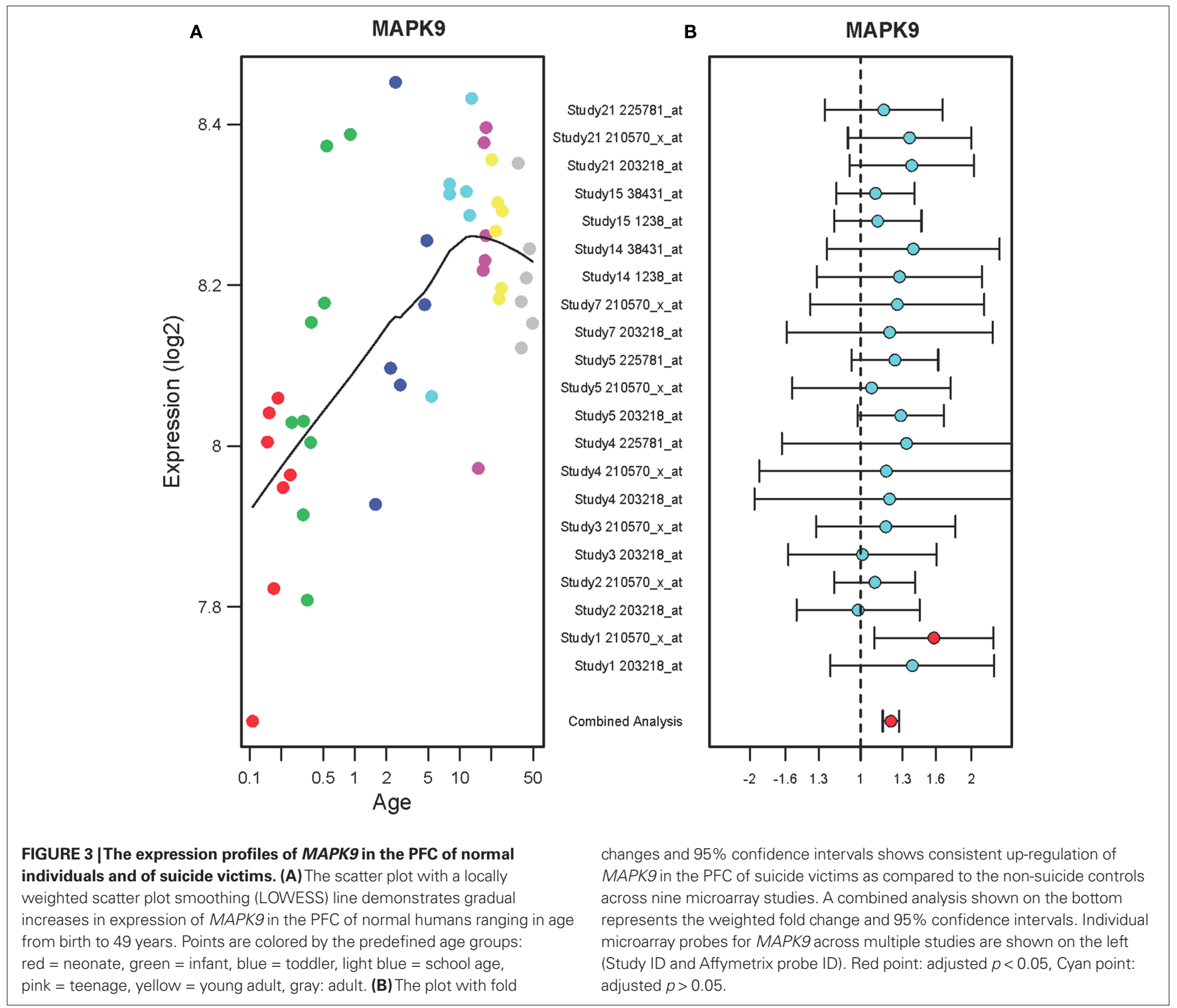

disorder. A recent study showed the advantage of performing the genetic analysis using only mood disorder subjects with or without suicide attempts (Perlis et al., 2010). Thus, we have taken a similar approach of comparing suicide vs. non-suicide among the mood disorder subjects. Because we used only mood disorder samples, we were able to minimize the potential confounding effects of comorbid psychiatric disorders in our analysis.

Although many kinases are involved in a complex network of intracellular phosphorylation, previous studies suggested that multiple kinase genes such as $P K C, C A M K, M A P K$, and $C D K$ play a significant role in regulating fear memories (Schafe et al., 2000; Li et al., 2002; Frankland et al., 2004; Lepicard et al., 2006; Sananbenesi et al., 2007; Bergstrom et al., 2011). CAMK2 including two splice variants, CaMK2A and CaMK2B, control dendritic growth and maturation in neurons, as well as phosphorylation of numerous receptors including GABAA receptor (Churn and Delorenzo, 1998) and NMDA glutamate receptor subunits (Rakic et al., 1994).
CAMK2 modulates catecholamine metabolism via phosphorylation of tyrosine hydroxylase in dopamine neurons (Seeman et al., 1976). Previous studies investigated the role of $C A M K 2 A$ and $C A M K 2 B$ in the postmortem brains of individuals with mood disorder. For example, a gene expression microarray study showed that CAMK2A expression levels were increased in the PFC of individuals with major depression (Tochigi et al., 2008). Another study replicated an up-regulation of $C A M K 2 A$ in the PFC of individuals with major depression but not bipolar disorder using a real-time quantitative PCR (Novak et al., 2006). The authors showed that the expression levels of $C a M K 2 A$ and $C A M K 2 B$ were elevated in the depression subjects by 29 and $36 \%$, respectively. Moreover, the increased levels in depression subjects were not altered by a history of antidepressant medication in that study. Here, we classified the mood disorder subjects into either the suicide or the non-suicide group and we had a slightly higher number of depression cases in the suicide group. Although the increased levels of $C A M K 2 B$ in the suicide 


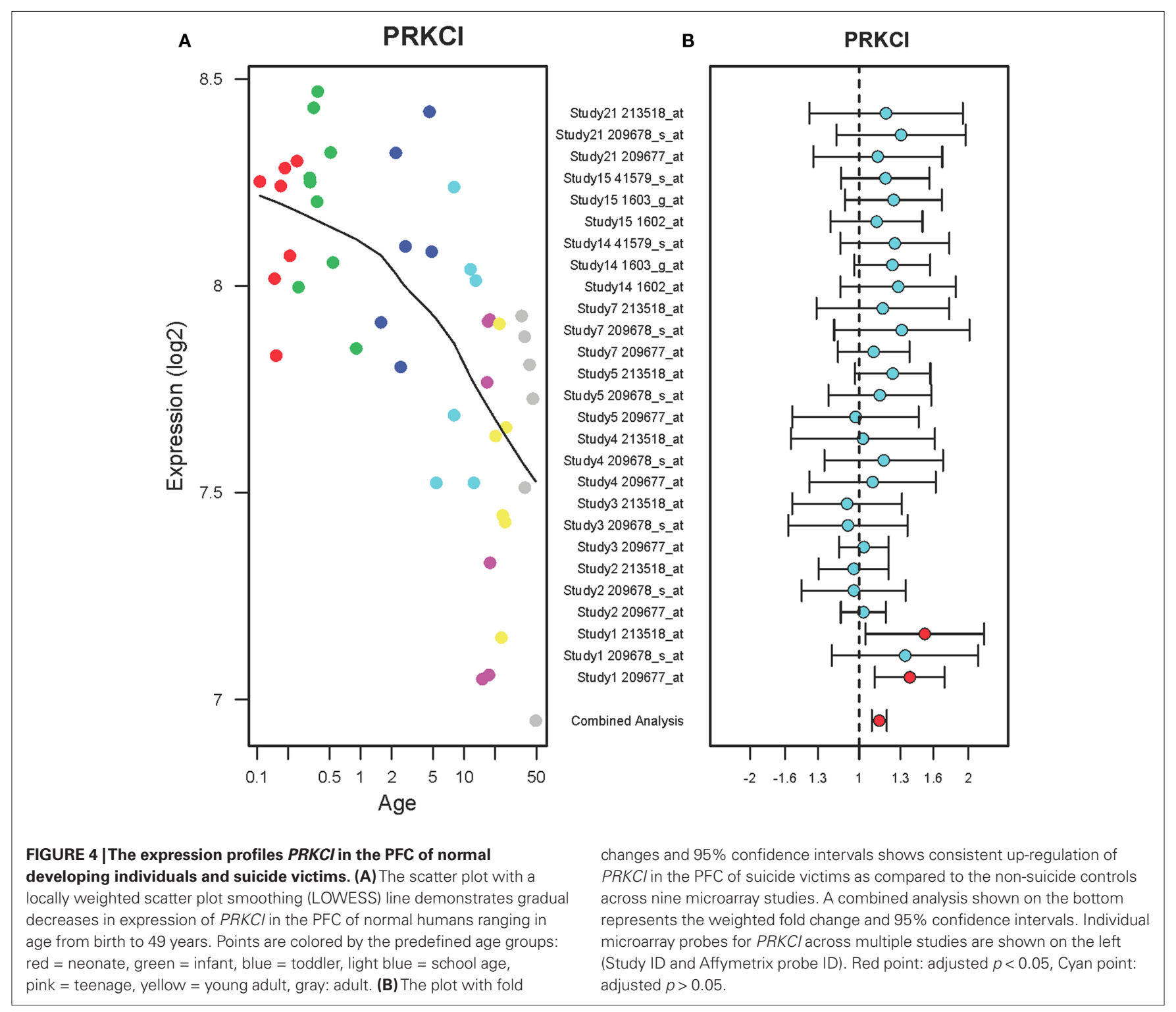

group may have been influenced by higher number of depression samples in the suicide group, this is unlikely because we adjusted for the mood disorder effects in the multiple regression analysis of suicide. Given that $C A M K 2 B$ could phosphorylate and influence the activity of many neurotransmitter receptors as well as neuronal growth and pruning, its altered expression in both developing and suicide brains suggests an important role in fear and stress.

CDK5 has been implicated in anxiety and stress-related disorders that may require the promotion of the fear extinction process, which is defined as the learned reduction of fear. Some of the roles of CDK5 during neurodevelopmental processes, such as interactions with distinct cytoplasmic and synaptic target molecules, may be related to the synaptic plasticity underlying memory consolidation (Fischer et al., 2003). A study using genetic and pharmacological approaches showed that extinction of fear memory requires the down-regulation of CDK5 in mice (Sananbenesi et al., 2007). The authors demonstrated that several key proteins associated with the
CDK5 pathway play a critical role in extinction of fear memory. Here, we found age-dependent changes in CDK5 expression: upregulation until 10 years of age and then gradual down-regulation until 49 years of age. This suggests that there is a sensitive period of CDK5 expression in the PFC during postnatal development. Thus, any disruptions in normal gene expression changes during development may result in dysfunction of stress and fear mechanisms. Increases in CDK5 levels in the PFC of suicide victims further suggest that fear learning and extinction mechanisms may be disrupted in the individuals with suicide.

$M A P K$ signaling pathway is critical for cell division and differentiation in the hippocampus as well as subsequent synaptic plasticity and memory formation (Sweatt, 2001). Importantly, $M A P K$ has been shown to be critical for the formation of new fear memories, as well as for extinction and reconsolidation of fear memories (Schafe et al., 2000; Herry et al., 2006; Bergstrom et al., 2011). These studies also support the role of neuronal plasticity 
in the PFC for fear extinction (Herry et al., 2006). Stress, acting via glucocorticoids, may directly interact with $M A P K$ mediated neuronal plasticity. A study showed that activation of glucocorticoid receptors increased the expression and enzymatic activity of proteins associated with the MAPK signaling pathway in mouse hippocampus (Revest et al., 2005). Inhibition of the MAPK pathway in the hippocampus abolished the increases in contextual fear conditioning induced by glucocorticoids. These results suggest a functional interaction between glucocorticoids receptors and the $M A P K$ system in fear learning and memory. An epigenetic study showed that $M A P K 1$ regulates hippocampal chromatin remodeling in memory formation (Chwang et al., 2007). The authors identified the mitogen- and stress-activated protein kinase 1 (MSK1), a nuclear kinase downstream of ERK, as an important regulator of chromatin remodeling in long-term memory formation. Here, we found that the expression levels of MAPK9 in the PFC of normal individuals gradually increased until 20 years of age then stabilized during postnatal development. This suggests that there is an increased demand on MAPK9 function in the PFC during the sensitive period of brain development. Moreover, abnormal increases in MAPK9 levels in the PFC of suicide victims indicate a critical role of this gene in the pathophysiology of suicide.

Previous studies suggested that the protein kinase C (PRKC) gene is involved in fear memory and suicide. For example, a chronic administration of $P R K C$ inhibitor staurosporine $(0.1 \mathrm{mg} / \mathrm{kg}$, for 14 days) significantly reduced the acquisition of conditioned fear in rats (Li et al., 2002). This supports the notion that PRKC plays a key role in synaptic plasticity underlying emotional learning and memory. However, it is important to note that the staurosporine is not a selective inhibitor of PRKC and therefore other kinases are also inhibited by this compound. A postmortem brain study examined potential association between the pathogenesis of teenage suicide and PRKC using 17 teenage suicide victims and 17 non-psychiatric control subjects (Pandey et al., 2004). Enzymatic activity, protein and mRNA levels of various PRKC isozymes (including $P R K C$ alpha, beta, and gamma) were measured in the PFC and hippocampus of suicide and non-suicide groups. There were significant decreases in protein and mRNA levels of PRKC alpha, beta, and gamma isozymes in the PFC and hippocampus of suicide victims as compared to the non-psychiatric controls. However, potential confounding effects of comorbid psychiatric disorders in the suicide victims have not been controlled in that study. Moreover, another PRKC isozyme, PRKC iota (PRKCI), was not measured. Here, we showed that the expression levels of $P R K C I$ in the PFC were gradually decreased across age from birth to 49 years of age and increased in the PFC of mood disorder patients with suicide as compared to the non-suicide mood disorder subjects. Consistent with our findings, a study demonstrated that inhibition of $P R K C$ signaling protected dendritic spines in the PFC and restored working memory impairment caused by chronic stress (Hains et al., 2009). This suggests that stress and anxiety may

\section{REFERENCES}

Bergstrom, H. C., Mcdonald, C. G., and Johnson, L. R. (2011). Pavlovian fear conditioning activates a common pattern of neurons in the lateral amygdala of individual brains. PLoS
ONE 6, e15698. doi: 10.1371/journal. pone.0015698

Brezo, J., Klempan, T., and Turecki, G. (2008). The genetics of suicide: a critical review of molecular studies. Psychiatr. Clin. North Am. 31, 179-203.

disrupt $P R K C$ signaling in the PFC and inhibition of $P R K C$ function may be neuroprotective and beneficial for the treatment of anxiety disorders. Many biological functions are mediated through phosphorylation by $P R K C$ in the brain and therefore, $P R K C$ may be a potential target for therapeutic intervention in individuals with anxiety disorders and suicidal ideation.

Postmortem brain studies of psychiatric patients are often challenging because many known and unknown factors can affect gene expression profiles in the brain tissue. Biological effects are often hindered by relatively small sample sizes, small effect sizes and comorbid psychiatric disorders. Available clinical information from each patient is typically sparse so that unknown covariates may either confound or confuse gene expression findings in postmortem brains. Thus, appropriate statistical adjustment is critical to improve inferences in determining gene expression changes in the brain of suicide victims. Although gene expression studies using postmortem brain tissue may reveal valuable information related to suicide, this approach alone is limited in terms of being able to distinguish between changes reflecting the primary disease etiology from those reflecting compensatory mechanisms and many potential confounding influences such as medication and substance use. Thus, it is important to study the functional role of these kinase genes that are being differentially expressed in the PFC of suicide victims as compared to the non-suicide subjects. In order to allow for a conclusive evaluation of the current findings, replication studies using independent samples as well as functional in vitro assays and pharmacological studies in animals are warranted. More research is needed to better understand the significance of the kinase genes that may be involved in the pathophysiology of suicide.

\section{CONCLUSION}

We identified four kinase genes including $C A M K 2 B, C D K 5$, $M A P K 9$, and PKCI that show both age-dependent changes in normal individuals as well as pathological changes in suicide victims. Importantly, these kinases are critical for fear and stress associated neuronal plasticity. Given that these genes play a critical role in the mechanisms of fear and stress, abnormal changes in expression in the PFC of suicide victims further suggest overlapping biological pathways between fear, stress, and suicide. Thus, any disruptions in normal expression changes during the sensitive period of postnatal development may result in dysfunction of those genes that contribute to the pathophysiology of suicide in adulthood. A better understanding of the kinase genes and their interaction with environmental factors may help to develop novel strategies for suicide prevention.

\section{ACKNOWLEDGMENTS}

We would like to thank the collaborators who contributed their microarray data for the cross-study analysis: Drs. Anthony Altar, Sabine Bahn, Haiming Chen, Seth Dobrin, Tadafumi Kato, Pamela Sklar, Curridium, Maree Webster, Cynthia Weickert, Svante Paabo.

Callahan, D. J. (2010). Combat-related mental health disorders: the case for resiliency in the long war. J. Am. Osteopath. Assoc. 110, 520-527.

Choi, K.H., Elashoff,M.,Higgs, B.W., Song, J., Kim, S., Sabunciyan, S., Diglisic, S.,
Yolken, R. H., Knable, M. B., Torrey, E. F., and Webster, M. J. (2008). Putative psychosis genes in the prefrontal cortex: combined analysis of gene expression microarrays. BMC Psychiatry 8, 87. doi: 10.1186/1471-244X-8-87 
Choi, K. H., Zepp, M. E., Higgs, B. W., Weickert, C. S., and Webster, M. J. (2009). Expression profiles of schizophrenia susceptibility genes during human prefrontal cortical development. J. Psychiatry Neurosci. 34, 450-458.

Churn, S. B., and Delorenzo, R. J. (1998). Modulation of GABAergic receptor binding by activation of calcium and calmodulin-dependent kinase II membrane phosphorylation. Brain Res. 809, 68-76.

Chwang, W. B., Arthur, J. S., Schumacher, A., and Sweatt, J. D. (2007). The nuclear kinase mitogen- and stressactivated protein kinase 1 regulates hippocampal chromatin remodeling in memory formation. J. Neurosci. 27, 12732-12742.

Conrad, C. D., Ledoux, J. E., Magarinos, A. M., and McEwen, B. S. (1999). Repeated restraint stress facilitates fear conditioning independently of causing hippocampal CA3 dendritic atrophy. Behav. Neurosci. 113, 902-913.

Fiori, L. M., Bureau, A., Labbe, A., Croteau, J., Noel, S., Merette, C., and Turecki, G. (2011). Global gene expression profiling of the polyamine system in suicide completers. Int. J. Neuropsychopharmacol. 14,595-605.

Fischer, A., Sananbenesi, F., Spiess, J., and Radulovic, J. (2003). Cdk5: a novel role in learning and memory. Neurosignals $12,200-208$.

Frankland, P. W., Bontempi, B., Talton, L. E., Kaczmarek, L., and Silva, A. J. (2004). The involvement of the anterior cingulate cortex in remote contextual fear memory. Science 304, 881-883.

Gentleman, R. C., Carey, V. J., Bates, D. M., Bolstad, B., Dettling, M., Dudoit, S., Ellis, B., Gautier, L., Ge, Y., Gentry, J., Hornik, K., Hothorn, T., Huber, W., Iacus, S., Irizarry, R., Leisch, F., Li, C., Maechler, M., Rossini, A. J., Sawitzki, G., Smith, C., Smyth, G., Tierney, L., Yang, J. Y., and Zhang, J. (2004). Bioconductor: open software development for computational biology and bioinformatics. Genome Biol. $5, \mathrm{R} 80$.

Guerra, V. S., and Calhoun, P. S. (2011). Examining the relation between posttraumatic stress disorder and suicidal ideation in an OEF/OIF veteran sample. J. Anxiety Disord. 25, 12-18.

Hains, A. B., Vu, M. A., Maciejewski, P. K., Van Dyck, C. H., Gottron, M., and Arnsten, A. F. (2009). Inhibition of protein kinase $\mathrm{C}$ signaling protects prefrontal cortex dendritic spines and cognition from the effects of chronic stress. Proc. Natl. Acad. Sci. U.S.A. 106, 17957-17962.
Harrison, P. J. (2011). Using our brains: the findings, flaws, and future of postmortem studies of psychiatric disorders. Biol. Psychiatry 69, 102-103.

Herry, C., Trifilieff, P., Micheau, J., Luthi, A., and Mons, N. (2006). Extinction of auditory fear conditioning requires MAPK/ERKactivation in the basolateral amygdala. Eur. J. Neurosci. 24, 261-269.

Irizarry, R. A., Hobbs, B., Collin, F., Beazer-Barclay, Y. D., Antonellis, K. J., Scherf, U., and Speed, T. P. (2003). Exploration, normalization, and summaries of high density oligonucleotide array probe level data. Biostatistics 4, 249-264.

Jakupcak, M., Cook, J., Imel, Z., Fontana, A., Rosenheck, R., and Mcfall, M. (2009). Posttraumatic stress disorder as a risk factor for suicidal ideation in Iraq and Afghanistan war veterans. J. Trauma Stress 22, 303-306.

Kim, J. J., and Diamond, D. M. (2002). The stressed hippocampus, synaptic plasticity and lost memories. Nat. Rev. Neurosci. 3, 453-462.

Kouzu, Y., Moriya, T., Takeshima, H., Yoshioka, T., and Shibata, S. (2000). Mutant mice lacking ryanodine receptor type 3 exhibit deficits of contextual fear conditioning and activation of calcium/calmodulin-dependent protein kinase II in the hippocampus. Brain Res. Mol. Brain Res. 76, 142-150.

Ledoux, J. E. (2000). Emotion circuits in the brain. Annu. Rev. Neurosci. 23, 155-184.

Lepicard, E. M., Mizuno, K., AntunesMartins, A., Von Hertzen, L. S., and Giese, K. P. (2006). An endogenous inhibitor of calcium/calmodulindependent kinase II is up-regulated during consolidation of fear memory. Eur. J. Neurosci. 23, 3063-3070.

Li, X. B., Inoue, T., and Koyama, T. (2002). Effect of chronic treatment with the protein kinase $\mathrm{C}$ inhibitor staurosporine on the acquisition and expression of contextual fear conditioning. Eur. J. Pharmacol. 441, 151-155.

Mann, J. J., Arango, V. A., Avenevoli, S., Brent, D. A., Champagne, F. A., Clayton, P., Currier, D., Dougherty, D. M., Haghighi, F., Hodge, S. E., Kleinman, J., Lehner, T., Mcmahon, F., Moscicki, E. K., Oquendo, M. A., Pandey, G. N., Pearson, J., Stanley, B., Terwilliger, J., and Wenzel, A. (2009). Candidate endophenotypes for genetic studies of suicidal behavior. Biol. Psychiatry 65, 556-563.

Mccullumsmith, R. E., and MeadorWoodruff, J. H. (2011). Novel approaches to the study of postmortem brain in psychiatric illness: old limitations and new challenges. Biol. Psychiatry 69, 127-133.

McEwen, B. S. (2007). Physiology and neurobiology of stress and adaptation: central role of the brain. Physiol. Rev. 87, 873-904.

Nepon, J., Belik, S. L., Bolton, J., and Sareen, J. (2010). The relationship between anxiety disorders and suicide attempts: findings from the National Epidemiologic Survey on Alcohol and Related Conditions. Depress. Anxiety 27, 791-798.

Novak, G., Seeman, P., and Tallerico, T. (2006).Increased expression of calcium/ calmodulin-dependent protein kinase IIbeta in frontal cortex in schizophrenia and depression. Synapse 59, 61-68.

Pandey, G. N., Dwivedi, Y., Rizavi, H. S., Ren, X., and Conley, R. R. (2004). Decreased catalytic activity and expression of protein kinase $\mathrm{C}$ isozymes in teenage suicide victims: a postmortem brain study. Arch. Gen Psychiatry 61, 685-693.

Perlis, R. H., Huang, J., Purcell, S., Fava, M., Rush, A. J., Sullivan, P. F., Hamilton, S. P., Mcmahon, F. J., Schulze, T. G. Potash, J. B., Zandi, P. P., Willour, V. L., Penninx, B. W., Boomsma, D. I., Vogelzangs, N., Middeldorp, C. M., Rietschel, M., Nothen, M., Cichon, S. Gurling, H., Bass, N., Mcquillin, A. Hamshere, M., Craddock, N., Sklar, P., and Smoller, J. W. (2010). Genomewide association study of suicide attempts in mood disorder patients. Am. J. Psychiatry 167, 1499-1507.

Rakic, P., Bourgeois, J. P., and GoldmanRakic, P. S. (1994). Synaptic development of the cerebral cortex: implications for learning, memory, and mental illness. Prog. Brain Res. 102, 227-243.

Revest, J. M., Di Blasi, F., Kitchener, P., Rouge-Pont, F., Desmedt, A., Turiault, M., Tronche, F., and Piazza, P. V. (2005). The MAPK pathway and Egr-1 mediate stress-related behavioral effects of glucocorticoids. Nat. Neurosci. 8, 664-672.

Rodrigues, S. M., Ledoux, J. E., and Sapolsky, R. M. (2009). The influence of stress hormones on fear circuitry. Annu. Rev. Neurosci. 32, 289-313.

Sananbenesi, F., Fischer, A., Wang, X. Schrick, C., Neve, R., Radulovic, J., and Tsai,L.H. (2007).A hippocampal Cdk5 pathway regulates extinction of contextual fear. Nat. Neurosci. 10, 1012-1019.

Schafe, G. E., Atkins, C. M., Swank, M. W. Bauer, E. P., Sweatt, J. D., and Ledoux, J. E. (2000). Activation of ERK/MAP kinase in the amygdala is required for memory consolidation of pavlovian fear conditioning. J. Neurosci. 20 8177-8187.

Seeman, P., Lee, T., Chau-Wong, M., and Wong, K. (1976). Antipsychotic drug doses and neuroleptic/dopamine receptors. Nature 261, 717-719.

Sweatt, J. D. (2001). The neuronal MAP kinase cascade: a biochemical signal integration system subserving synaptic plasticity and memory. $J$. Neurochem. 76, 1-10.

Tarrier, N., and Picken, A. (2010). Co-morbid PTSD and suicidality in individuals with schizophrenia and substance and alcohol abuse. Soc. Psychiatry Psychiatr. Epidemiol. doi: 10.1007/s00127-010-0277-0. [Epub ahead of print].

Tochigi, M., Iwamoto, K., Bundo, M., Sasaki, T., Kato, N., and Kato, T. (2008). Gene expression profiling of major depression and suicide in the prefrontal cortex of postmortem brains. Neurosci. Res. 60, 184-191.

Torrey, E. F., Webster, M., Knable, M., Johnston, N., and Yolken, R. H. (2000). The stanley foundation brain collection and neuropathology consortium. Schizophr. Res. 44, 151-155.

Tsai, S. J., Hong, C. J., and Liou, Y.J. (2011). Recent molecular genetic studies and methodological issues in suicide research. Prog. Neuropsychopharmacol. Biol. Psychiatry 35, 809-817.

Ursano, R. J., Goldenberg, M., Zhang, L., Carlton, J., Fullerton, C. S., Li, H., Johnson, L., and Benedek, D. (2010). Posttraumatic stress disorder and traumatic stress: from bench to bedside, from war to disaster. Ann. N. Y. Acad. Sci. 1208, 72-81.

Conflict of Interest Statement: The authors declare that the research was conducted in the absence of any commercial or financial relationships that could be construed as a potential conflict of interest.

Received: 31 May 2011; paperpending published: 16 June 2011; accepted: 16 Jul 2011; published online: 28 July 2011.

Citation: Choi K, Le T, Xing G, Johnson LR and Ursano RJ (2011) Analysis of kinase gene expression in the frontal cortex of suicide victims: implications offear and stress. Front. Behav. Neurosci. 5:46. doi: 10.3389/ fnbeh.2011.00046

Copyright $(2011$ Choi, Le, Xing, Johnson and Ursano. This is an open-access article subject to a non-exclusive license between the authors and Frontiers Media SA, which permits use, distribution and reproduction in other forums, provided the original authors and source are credited and other Frontiers conditions are complied with. 